\title{
Metal tolerance analysis of Gram negative bacteria from hospital effluents of Northern India
}

\author{
Manzar Alam*, Mohd Imran \\ Department of Biosciences, Integral University, Lucknow, India.
}

\begin{tabular}{|c|c|}
\hline ARTICLE INFO & ABSTRACT \\
\hline Article history: & \multirow{11}{*}{$\begin{array}{l}\text { Effluents from different hospitals were analysed to Nickel, Chromium, Cobalt, Copper, Mercury, Cadmium and } \\
\text { Zinc resistance among Gram negative bacteria. The resistance among the Gram negative bacterial population } \\
\text { varied considerably in different metal and water sampling sites. Gram negative bacteria showed lower metal } \\
\text { resistant viable count range } 4.01 \times 10^{4}-1.3 \times 10^{3} \text { at } 50-100 \mu \mathrm{g} / \mathrm{ml} \text { in site-IV as compared to } 11.03 \times 105-1.03 \times 10^{4} \text {, } \\
12.02 \times 10^{5}-1.4 \times 10^{3} \text { and } 12.33 \times 10^{5}-2.7 \times 10^{3} \text { in site-I, II and III against all metal tested, respectively. Viable } \\
\text { counts of Gram negative bacterial population were recorded higher against Nickel and Zinc from sampling site- } \\
\text { III as compared to other sites tested. Lower viable counts of Gram negative bacteria were recorded against } \\
\text { Mercury in all sites tested. All the isolates of Gram negative bacteria showed their tolerance level (Minimum } \\
\text { inhibitory concentration) in the range of } 50-1600 \mu \mathrm{g} / \mathrm{ml} \text { against all the metal tested. Of } 88 \% \text {, } 76 \% \text { and } 86 \% \\
\text { isolates exhibited their MIC at } 50-100 \mu \mathrm{g} / \mathrm{ml} \text { against Mercury, Cadmium and Cobalt in all the sites tested, } \\
\text { respectively. Maximum } 60 \% \text { and } 32 \% \text { of the isolates demonstrated their MIC at } 1200-1600 \mu \mathrm{g} / \mathrm{ml} \text { against } \mathrm{Cr}{ }^{2+} \\
\text { and } \mathrm{Cu}^{2+} \text { from the entire site tested, respectively. All Gram negative bacterial isolates also observed multiple } \\
\text { resistance patterns }(2-7 \text { metal) in different combination of metals. The Multi metal resistance Index (MMR) } \\
\text { index ranges were found }(0.03-0.71) \text { indicating the high risk of environmental contamination and emergence of } \\
\text { metal resistance which may promote the development of resistance to antibiotics among the pathogens. }\end{array}$} \\
\hline $\operatorname{Re}$ & \\
\hline $\mathrm{Ac}$ & \\
\hline Available & \\
\hline Key & \\
\hline Gram negative Bacteria, & \\
\hline Hospital Wastewater, & \\
\hline Minimum inhibitory & \\
\hline $\begin{array}{l}\text { concentration, Multi metal } \\
\text { resistance Index, Viable }\end{array}$ & \\
\hline & \\
\hline & \\
\hline
\end{tabular}

\section{INTRODUCTION}

Wastewater released from hospitals could be loaded with antimicrobial resistant micro-organism and toxic chemicals. Improperly treated hospital wastewater is hazardous for reuse or for releasing into natural water source (Rutala and Mayhall, 1992; Blumenthal et al., 2001). Metal pollution remains a major challenge in environmental biotechnology. Some industrial processes results in the discharge of metals into aquatic systems. The concentration of metal pollutants in the environment is usually low excluding in specific areas, which are polluted by various hospitals and industrial wastes. The concentration of heavy metals is very high in ore containing and mining areas (Roane et al., 1996). This has led to growing concern about the consequence of toxic metals as environmental pollutants. This kind of contamination presents a challenge, as the presence of

* Corresponding Author

Email:manzar.160@gmail.com metals in soils and aqueous effluents leads to severe trouble because they cannot be biodegraded. Unlike many other pollutants, metals are complicated to remove from the environment (Ren et al., 2009). Some heavy metals such as nickel, iron, copper and zinc are necessary to metabolic reactions and are required as trace elements by the organisms. Others like mercury, silver and cadmium have no biological role and are injurious to the organisms, even at very low concentrations (Hughes et al., 1989). Many bacteria have precise genetic mechanisms of resistance to toxic metals (Silver and Misra, 1988; Mindlin et al., 2001). In the environment metals, may select these resistant variants in a manner similar to the selection of antibiotic resistant strains. Indeed, it is relatively frequent the association of metal and antimicrobial resistance, since both resistance genes are commonly located on the same mobile genetic elements (Foster, 1983; McIntosh et al., 2008). Accordingly, it can be assumed that the selective pressure exerted by heavy metals contribute to the indirect co-selection of antibiotic resistance, particularly in environments polluted with the two elements. 
Microorganisms resistant to both antibiotics and metals have been isolated commonly from the environments, and this has led to proposition that the combined expression of antibiotic resistance \& metal resistance is caused by selection, consequential from metals present in particular environment (Bell et al., 1983; Viti and Giovannetti, 2003). The occurrence of antibiotic-resistant bacteria in the natural habitats can pose a public health risk (Nies, 1999). The study was aimed to explore the threat of wastewater generated from hospitals to the public. Wastewater treatment requires suitable methods and constant monitoring to ensure that the treated effluent be not hazardous to the environment.

\section{MATERIAL AND METHODS}

\section{Sample collection}

Water samples were collected from three sites of hospital wastewater along with King George's Medical University Site -I untreated, Sanjay Gandhi Post Graduate Institute of Medical Sciences Site-II treated, Sanjay Gandhi Post Graduate Institute of Medical Sciences Site-III untreated and Dr. Ram Manohar Lohia Hospital Site -IV untreated at Lucknow city as shown in Figure 1. Samples were collected in sterile 250-ml polypropylene bottles, according to internationally recommended methodology (Lösch et al., 2008). Samples were kept at $4^{\circ} \mathrm{C}$ until their arrival to laboratory.

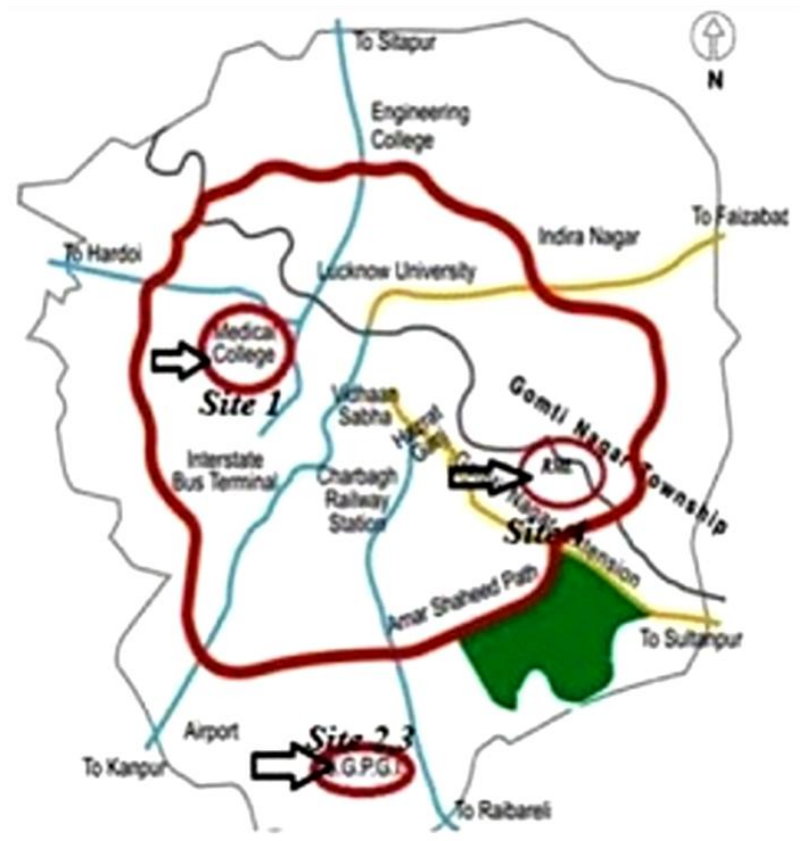

Fig. 1: Sampling sites.

\section{Isolation and identification of metal tolerant Gram negative bacterial population}

Isolation of metal resistant Gram negative bacteria from water samples were done on metal amended Mac conky agar plates at varying concentration $(25-1200 \mu \mathrm{g} / \mathrm{ml})$. Serial dilutions of the water samples were plated by spreading $0.1 \mathrm{ml}$ on medium for the count of total metal resistant Gram negative bacteria. Plates incubated at $37^{\circ} \mathrm{C}$ for 24 hours and Gram negative bacterial counts were expressed as CFU/ml on Mac conky agar medium. The selected isolates were finally identified on the basis of biochemical characterization as described elsewhere (Cappuccino and Sherman, 1995).

\section{Determination of minimum inhibitory concentration (MIC) of metal among different Gram negative isolates}

The heavy metal resistance was determined by the minimum inhibitory concentration (MIC) against the test bacterial strain by spot plate method (Malik and Jaiswal, 2000). Nutrient plates of each heavy metal (Chromium K2Cr2O7, Cadmium $\mathrm{CdCl} 2$, Cobalt $\mathrm{CoCl} 2$, Mercury $\mathrm{HgCl} 2$, Copper $\mathrm{CuSo} 4$, Zinc $\mathrm{ZnCl} 2$ and Nickel $\mathrm{NiCl} 2)$ of different concentrations $(50 \mu \mathrm{g} / \mathrm{ml}$ to $1600 \mu \mathrm{g} / \mathrm{ml})$ were prepared. Inoculums of test strain $(3 \times 106$ $\mathrm{CFU} / \mathrm{ml}$ ) were spotted on heavy metal amended plates and control plates in duplicate with the help of platinum loop of $5 \mathrm{~mm}$ diameter.

The plates were incubated at $37^{\circ} \mathrm{C}$ for $24 \mathrm{hr}$ to observe the growth of bacterial strain on the spotted area. The MIC was defined as the minimum inhibitory concentration of the heavy metal that inhibits the visible growth of test strain. Metal concentration range bellow MIC was considered as sub MIC of the isolates.

\section{Multiple metal resistances (MMR) indexing}

The MMR index profile based on isolate was evaluated to access the health risk of the environment. MMR index for test isolates was calculated according to the formula: No. of metal to which all isolates were resistant/No. of metal tested $x$ No. of isolates.

\section{RESULTS}

In this study, metal tolerant population of gram negative bacteria from the hospital waste water was observed against seven heavy metal $\left(\mathrm{Hg}^{2+}, \mathrm{Cd}^{2+}, \mathrm{Cu}^{2+}, \mathrm{Zn}^{2+}, \mathrm{Ni}^{2+}, \mathrm{Co}^{2+}\right.$ and $\left.\mathrm{Cr}^{3+}\right)$ at their varying concentrations $(25-1200 \mu \mathrm{g} / \mathrm{ml})$. Viable $(\mathrm{CFU} / \mathrm{ml})$ count of gram negative bacteria was observed higher in (non-metal supplemented) control plate than metal supplemented plates in all the sites tested. The viable count of Gram negative bacteria in different concentrations of metal ranged from $11.03 \times 10^{5}-8.0 \times 10^{2}$, $12.02 \times 10^{5}-1.0 \times 10^{2}, \quad 12.33 \times 10^{5}-5.0 \times 10^{2}$ and $4.01 \times 10^{4}-1.0 \times 10^{2}$ $\mathrm{cfu} / \mathrm{ml}$ of water in site I, II, III and IV, respectively. Maximum viable count was observed against $\mathrm{Cu}^{2+}$ and $\mathrm{Cr}^{3+}$ in sampling site-I, while the same was found against $\mathrm{Co}^{2+}$ and $\mathrm{Ni}^{2+}$ in site II, III at $25 \mu \mathrm{g} / \mathrm{ml}$ respectively. In site IV maximum viable count were observed against $\mathrm{Zn}^{2+}$. Minimum viable count was observed against $\mathrm{Hg}^{2+}$ and $\mathrm{Cd}^{2+}$ at $25 \mu \mathrm{g} / \mathrm{ml}$ in sites I, II and IV, respectively. While in site-III minimum viable counts were observed $\mathrm{Hg}^{2+}$ and $\mathrm{Cu}^{2+}$ at $25 \mu \mathrm{g} / \mathrm{ml}$ concentration. Maximum number of viable count was recorded at higher concentration against $\mathrm{Cu}^{2+}\left(1.06 \times 10^{4}\right)$ and $\mathrm{Zn}^{2}+\left(4.35 \times 10^{4}\right)$ in site II and III, respectively (Table1). 
Table 1: Viable count of metal tolerant Gram negative bacteria from different wastewater sampling sites.

\begin{tabular}{|c|c|c|c|c|c|}
\hline Metal & Conc. & Site1 & Site2 & Site3 & Site4 \\
\hline Control & No metal & $12.5 \times 10^{5} \pm 0.56$ & $13.00 \times 10^{5} \pm 1.19$ & $13.43 \times 10^{5} \pm 1.19$ & $8.13 \times 10^{4} \pm 0.65$ \\
\hline \multirow{6}{*}{$\mathrm{Hg}^{2+}$} & 25 & $2.9 \times 10^{3} \pm 0.01$ & $1.4 \times 10^{3} \pm 0.11$ & $2.7 \times 10^{3} \pm 0.4$ & $1.3 \times 10^{3} \pm 0.13$ \\
\hline & 50 & $2.0 \times 10^{3} \pm 0.02$ & $4 \times 10^{2} \pm 0.41$ & $2.2 \times 10^{3} \pm 0.15$ & $7 \times 10^{2} \pm 0.55$ \\
\hline & 100 & $6.0 \times 10^{2} \pm 0.011$ & $3 \times 10^{2} \pm 0.32$ & $1.9 \times 10^{3} \pm 0.09$ & $1 \times 10^{2} \pm 0.11$ \\
\hline & 200 & ND & ND & ND & ND \\
\hline & 400 & ND & ND & ND & ND \\
\hline & 800 & ND & ND & ND & ND \\
\hline \multirow{7}{*}{$\mathrm{Cd}^{2+}$} & 25 & $3.9 \times 10^{3} \pm 0.02$ & $1.13 \times 10^{4} \pm 0.08$ & $5.22 \times 10^{4} \pm 0.56$ & $1.09 \times 10^{4} \pm 0.42$ \\
\hline & 50 & $2.7 \times 10^{3} \pm 0.04$ & $8.3 \times 10^{3} \pm 0.03$ & $4.32 \times 10^{4} \pm 0.32$ & $6.3 \times 10^{3} \pm 0.83$ \\
\hline & 100 & $1.2 \times 10^{3} \pm 0.09$ & $3.4 \times 10^{3} \pm 0.12$ & $4.09 \times 10^{4} \pm 0.09$ & $2.3 \times 10^{3} \pm 0.11$ \\
\hline & 200 & ND & $1.8 \times 10^{3} \pm 0.18$ & $3.89 \times 10^{4} \pm 0.17$ & $5 \times 10^{2} \pm 0.54$ \\
\hline & 400 & ND & ND & $1.69 \times 10^{4} \pm 0.12$ & $1 \times 10^{2} \pm 0.09$ \\
\hline & 800 & ND & ND & $6 \times 10^{2} \pm 0.65$ & ND \\
\hline & 1200 & ND & ND & ND & ND \\
\hline \multirow{7}{*}{$\mathrm{Co}^{2+}$} & 25 & $2.65 \times 10^{4} \pm 0.02$ & $11.03 \times 10^{5} \pm 1.19$ & $11.22 \times 10^{5} \pm 2.12$ & $3.23 \times 10^{4} \pm 0.13$ \\
\hline & 50 & $1.52 \times 10^{4} \pm 0.01$ & $9.35 \times 10^{4} \pm 0, .59$ & $10.13 \times 10^{5} \pm 1.97$ & $3.00 \times 10^{4} \pm 0.19$ \\
\hline & 100 & $9 \times 10^{2} \pm 0.09$ & $5.03 \times 10^{4} \pm 0.67$ & $9.15 \times 10^{4} \pm 0.57$ & $1.88 \times 10^{4} \pm 0.09$ \\
\hline & 200 & ND & $4.17 \times 10^{4} \pm 0.31$ & $8.23 \times 10^{4} \pm 0.69$ & $1.50 \times 10^{4} \pm 0.05$ \\
\hline & 400 & ND & $1.09 \times 10^{4} \pm 0.03$ & $7.93 \times 10^{4} \pm 0.54$ & $1.21 \times 10^{4} \pm 0.05$ \\
\hline & 800 & ND & ND & $5.02 \times 10^{4} \pm 0.65$ & $8.9 \times 10^{3} \pm 0.92$ \\
\hline & 1200 & ND & ND & $5 \times 10^{2} \pm 0.01$ & ND \\
\hline \multirow{7}{*}{$\mathrm{Ni}^{3+}$} & 25 & $1.55 \times 10^{4} \pm 0.12$ & $12.02 \times 10^{5} \pm 1.4$ & $12.33 \times 10^{5} \pm 1.09$ & $2.27 \times 10^{4} \pm 0.14$ \\
\hline & 50 & $1.23 \times 10^{4} \pm 0.14$ & $11.12 \times 10^{5} \pm 1.01$ & $12.01 \times 10^{5} \pm 1.09$ & $2.03 \times 10^{4} \pm 0.32$ \\
\hline & 100 & $9 \times 10^{2} \pm 0.19$ & $2.93 \times 10^{4} \pm 0.03$ & $9.53 \times 10^{4} \pm 0.97$ & $4.9 \times 10^{3} \pm 0.29$ \\
\hline & 200 & ND & $1.42 \times 10^{4} \pm 0.09$ & $8.97 \times 10^{4} \pm 0.45$ & $3.7 \times 10^{3} \pm 0.13$ \\
\hline & 400 & ND & $4.0 \times 10^{3} \pm 0.52$ & $6.13 \times 10^{4} \pm 0.32$ & $3 \times 10^{2} \pm 0.15$ \\
\hline & 800 & ND & ND & $4.22 \times 10^{4} \pm 0.54$ & $1 \times 10^{2} \pm 0.09$ \\
\hline & 1200 & ND & ND & $1.65 \times 10^{4} \pm 0.08$ & ND \\
\hline \multirow{7}{*}{$\mathrm{Zn}^{2+}$} & 25 & $1.03 \times 10^{4} \pm 0.02$ & $3.23 \times 10^{4} \pm 0.31$ & $12.8 \times 10^{5} \pm 1.29$ & $4.01 \times 10^{4} \pm 0.32$ \\
\hline & 50 & $8.3 \times 10^{3} \pm 0.13$ & $2.93 \times 10^{4} \pm 0.21$ & $11.93 \times 10^{5} \pm 1.00$ & $3.86 \times 10^{4} \pm 0.12$ \\
\hline & 100 & $6.9 \times 10^{3} \pm 0.09$ & $2.9 \times 10^{3} \pm 0.12$ & $11.13 \times 10^{5} \pm 1.41$ & $2.09 \times 10^{4} \pm 0.45$ \\
\hline & 200 & $4.5 \times 10^{3} \pm 0.06$ & $2.0 \times 10^{3} \pm 0.09$ & $8.65 \times 10^{4} \pm 0.80$ & $1.00 \times 10^{4} \pm 0.11$ \\
\hline & 400 & $1.3 \times 10^{3} \pm 0.02$ & $2 \times 10^{2} \pm 0.11$ & $8.48 \times 10^{4} \pm 0.66$ & $6.5 \times 10^{3} \pm 0.59$ \\
\hline & 800 & ND & $1 \times 10^{2} \pm 0.01$ & $6.15 \times 10^{4} \pm 0.50$ & $2.6 \times 10^{3} \pm 0.16$ \\
\hline & 1200 & ND & ND & $4.35 \times 10^{4} \pm 0.95$ & $1.8 \times 10^{3} \pm 0.09$ \\
\hline \multirow{7}{*}{$\mathrm{Cr}^{6+}$} & 25 & $9.35 \times 10^{4} \pm 0.11$ & $2.22 \times 10^{4} \pm 0.21$ & $5.23 \times 10^{4} \pm 0.39$ & $3.81 \times 10^{4} \pm 0.15$ \\
\hline & 50 & $3.48 \times 10^{4} \pm 0.05$ & $1.40 \times 10^{4} \pm 0.31$ & $4.07 \times 10^{4} \pm 0.14$ & $3.04 \times 10^{4} \pm 0.32$ \\
\hline & 100 & $2.09 \times 10^{4} \pm 0.01$ & $1.10 \times 10^{4} \pm 0.071$ & $3.69 \times 10^{4} \pm 0.12$ & $9.6 \times 10^{3} \pm 0.96$ \\
\hline & 200 & ND & $9.8 \times 10^{3} \pm 0.95$ & $1.93 \times 10^{4} \pm 0.12$ & $\begin{array}{l}2.3 \times 10^{3} \pm 0.14 \\
1.0 \times 10^{3}+0.01\end{array}$ \\
\hline & 400 & ND & $7 \times 10^{2} \pm 0.829$ & $9.2 \times 10^{3} \pm 0.96$ & $1.0 \times 10^{\circ} \pm 0.01$ \\
\hline & 800 & ND & ND & ND & ND \\
\hline & 1200 & ND & ND & ND & ND \\
\hline \multirow{7}{*}{$\mathrm{Cu}^{2+}$} & 25 & $11.03 \times 10^{5} \pm 0.15$ & $5.38 \times 10^{4} \pm 0.57$ & $4.05 \times 10^{4} \pm 0.03$ & $1.29 \times 10^{4} \pm 0.4$ \\
\hline & 50 & $3.09 \times 10^{4} \pm 0.11$ & $4.13 \times 10^{4} \pm 1.01$ & $3.06 \times 10^{4} \pm 0.42$ & $1.13 \times 10^{4} \pm 0.19$ \\
\hline & 100 & $2.35 \times 10^{4} \pm 0.09$ & $3.67 \times 10^{4} \pm 0.75$ & $2.11 \times 10^{4} \pm 0.12$ & $9.5 \times 10^{3} \pm 0.87$ \\
\hline & 200 & $1.88 \times 10^{4} \pm 0.03$ & $2.91 \times 10^{4} \pm 0.02$ & $2.03 \times 10^{4} \pm 0.11$ & $8.8 \times 10^{3} \pm 0.34$ \\
\hline & 400 & $1.03 \times 10^{4} \pm 0.01$ & $2.07 \times 10^{4} \pm 0.11$ & $1.67 \times 10^{4} \pm 0.22$ & $6.0 \times 10^{3} \pm 0.96$ \\
\hline & 800 & $8 \times 10^{2} \pm 0.29$ & $1.69 \times 10^{4} \pm 0.10$ & $1.39 \times 10^{4} \pm 0.02$ & $2.9 \times 10^{3} \pm 0.14$ \\
\hline & 1200 & ND & $1.06 \times 10^{4} \pm 0.09$ & $1.26 \times 10^{4} \pm 0.07$ & ND \\
\hline
\end{tabular}

Table 2: Multi-metal resistance pattern in 50 gram negative bacterial isolates from KGMU hospital (Untreated).

\begin{tabular}{|c|c|c|c|c|}
\hline No of Metal & Resistance Pattern & Isolates & $\begin{array}{ll}\text { Percentage } \%) \\
\end{array}$ & MMR Index \\
\hline 3 & $\mathrm{Cu}, \mathrm{Ni}, \mathrm{Zn}$ & 1 & 2 & 0.42 \\
\hline \multirow{4}{*}{4} & $\mathrm{Cr}, \mathrm{Cu}, \mathrm{Ni}, \mathrm{Zn}$ & 1 & \multirow{4}{*}{8} & \multirow{4}{*}{0.14} \\
\hline & $\mathrm{Co}, \mathrm{Ni}, \mathrm{Cd}, \mathrm{Zn}$ & 1 & & \\
\hline & $\mathrm{Cu}, \mathrm{Ni}, \mathrm{Cd}, \mathrm{Zn}$ & 1 & & \\
\hline & $\mathrm{Cr}, \mathrm{Cu}, \mathrm{Cd}, \mathrm{Zn}$ & 1 & & \\
\hline \multirow{5}{*}{5} & $\mathrm{Co}, \mathrm{Cu}, \mathrm{Ni}, \mathrm{Cd}, \mathrm{Zn}$ & 2 & \multirow{5}{*}{38} & \multirow{5}{*}{0.03} \\
\hline & Co. $\mathrm{Cr}, \mathrm{Cu}, \mathrm{Ni}, \mathrm{Zn}$ & 11 & & \\
\hline & $\mathrm{Hg}, \mathrm{Cr}, \mathrm{Cu}, \mathrm{Ni}, \mathrm{Cd}$ & 1 & & \\
\hline & $\mathrm{Hg}, \mathrm{Cr}, \mathrm{Cu}, \mathrm{Cd}, \mathrm{Zn}$ & 1 & & \\
\hline & $\mathrm{Cr}, \mathrm{Cu}, \mathrm{Ni}, \mathrm{Cd}, \mathrm{Zn}$ & 4 & & \\
\hline \multirow{5}{*}{6} & $\mathrm{Hg}, \mathrm{Co}, \mathrm{Cr}, \mathrm{Cu}, \mathrm{Ni}, \mathrm{Zn}$ & 4 & \multirow{5}{*}{42} & \multirow{5}{*}{0.04} \\
\hline & $\mathrm{Co}, \mathrm{Cr}, \mathrm{Cu}, \mathrm{Ni}, \mathrm{Cd}, \mathrm{Zn}$ & 13 & & \\
\hline & $\mathrm{Hg}, \mathrm{Co}, \mathrm{Cu}, \mathrm{Ni}, \mathrm{Cd}, \mathrm{Zn}$ & 1 & & \\
\hline & $\mathrm{Hg}, \mathrm{Co}, \mathrm{Cr}, \mathrm{Cu}, \mathrm{Ni}, \mathrm{Cd}$ & 1 & & \\
\hline & $\mathrm{Hg}, \mathrm{Cr}, \mathrm{Cu}, \mathrm{Ni}, \mathrm{Cd}, \mathrm{Zn}$ & 2 & & \\
\hline 7 & $\mathrm{Hg}, \mathrm{Co}, \mathrm{Cr}, \mathrm{Cu}, \mathrm{Ni}, \mathrm{Cd}, \mathrm{Zn}$ & 5 & 10 & 0.2 \\
\hline
\end{tabular}


All isolates were identified and characterized on the basis of their biochemical properties. The main genus were identified as 'E.coli, Enterobactor, Pseudomonas, Klebsiella, Salmonella, Serratia, Citrobacter and Proteus' from the entire sampling sites of hospital wastewater (Table 2) and then tested for their MIC against seven heavy metals $\left(\mathrm{Hg}^{2+}, \mathrm{Cd}^{2+}, \mathrm{Cu}^{2+}, \mathrm{Zn}^{2+}, \mathrm{Ni}^{2+}, \mathrm{Co}^{2+}\right.$ and $\left.\mathrm{Cr}^{3+}\right)$ at varying concentration $(50-1200 \mu \mathrm{g} / \mathrm{ml})$. The $\mathrm{Hg} 2+$ showed highest toxicity against all the gram-negative bacterial isolates from the entire sites tested. In site $1,72 \%$ of the total isolates showed their MIC range $50-100 \mu \mathrm{g} / \mathrm{ml}$ for $\mathrm{Hg} 2+$ followed by $42 \%$, $34 \%, 10 \%, 6 \%$ and $2 \%$ against $\mathrm{Cd}^{2+}, \mathrm{Co}^{2+}, \mathrm{Cr}^{3+}, \mathrm{Ni}^{2+}$ and $\mathrm{Zn}^{2+}$, respectively. Maximum $30 \%$ of the isolates showed their MIC range $1200-1600 \mu \mathrm{g} / \mathrm{ml}$ against $\mathrm{Cu}^{2+}$. No MIC range was recorded at lower concentration (50-100) against $\mathrm{Cu}^{2+}$ and at higher concentration (1200-1600) against $\mathrm{Hg}^{2+}, \mathrm{Cd}^{2+}, \mathrm{Co}^{2+}, \mathrm{Ni}^{2+}, \mathrm{Zn}^{2+}$ and $\mathrm{Cr}^{3+}$, respectively (Fig-2).

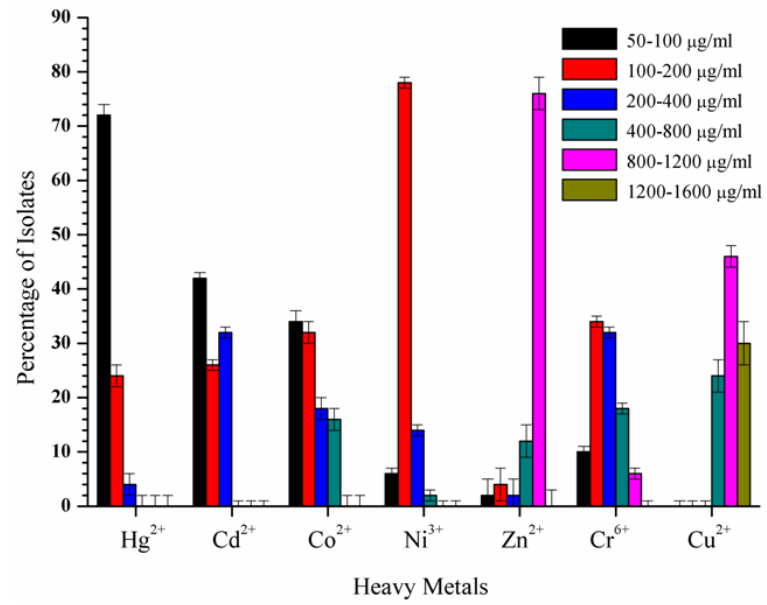

Fig. 2: Gram negative isolates showing various ranges of MIC of metal from site 1).

In site-II maximum $88 \%, 86 \%$ and $42 \%$ isolates showed their $\mathrm{MIC}$ range $50-100 \mu \mathrm{g} / \mathrm{ml}$ against $\mathrm{Hg}^{2+}, \mathrm{Co}^{2+}$, and $\mathrm{Cd}^{2+}$, respectively. $78 \%$ of the isolates showed their MIC range 100-200 $\mu \mathrm{g} / \mathrm{ml}$ against $\mathrm{Zn}^{2+}$ while $60 \%$, of the isolates demonstrated their MIC $1200-1600 \mu \mathrm{g} / \mathrm{ml}$ against $\mathrm{Cr}^{3+}$. The MIC was not detected at range 100-200 and, 400-800 $\mu \mathrm{g} / \mathrm{ml}$ against $\mathrm{Cr}^{3+}$, and $\mathrm{Hg}^{2+}, \mathrm{Cd}^{2+}$, $\mathrm{Co}^{2+}, \mathrm{Ni}^{2+}, \mathrm{Zn}^{2+}$ respectively (Fig-3).

In site-III, $76 \%$ of the isolates showed their MIC range $50-100 \mu \mathrm{g} / \mathrm{ml}$ against $\mathrm{Cd}^{2+}$ followed by $48 \%, 24 \%, 22 \%$ and $14 \%$ against $\mathrm{Hg}^{2+}, \mathrm{Zn}^{2+}, \mathrm{Cr}^{3+}, \mathrm{Co}^{2+}$ and $\mathrm{Cu}^{2+}$, respectively. Maximum $62 \%, 38 \%, 32 \%, 26 \%, 20 \%, 16 \%$ and $8 \%$ of the isolates showed their MIC (200-400 $\mu \mathrm{g} / \mathrm{ml})$ against $\mathrm{Cu}^{2+}, \mathrm{Ni}^{2+}, \mathrm{Zn}^{2+}, \mathrm{Cr}^{3+}, \mathrm{Co}^{2+}$, $\mathrm{Cd}^{2+}$ and $\mathrm{Hg}^{2+}$, respectively. No MIC was recorded at varying concentration range of the heavy metal tested (Fig-4).

In case of site-IV, no MIC was observed at range 50$100 \mu \mathrm{g} / \mathrm{ml}$ against $\mathrm{Co}^{2+}, \mathrm{Ni}^{2+}, \mathrm{Cr}^{3+}$ and $\mathrm{Cu}^{2+}$, respectively. $76 \%$ of the isolates showed their MIC at lower concentration (50-100 $\mu \mathrm{g} / \mathrm{ml}$ ) against $\mathrm{Hg}^{2+}$ while $46 \%$ of the isolates showed their MIC at $1200-1600 \mu \mathrm{g} / \mathrm{ml}$ against $\mathrm{Cr}^{3+}$. Maximum $72 \%, 66 \%, 42 \%, 38 \%$, $34 \%, 16 \%$ and $12 \%$ of the isolates showed their MIC at $200-400$ $\mu \mathrm{g} / \mathrm{ml}$ against $\mathrm{Zn}^{2+}, \mathrm{Co}^{2+} \mathrm{Cu}^{2+}, \mathrm{Cd}^{2+}, \mathrm{Ni}^{2+}, \mathrm{Hg}^{2+}$ and $\mathrm{Cr}^{3+}$, respectively (Fig-5).

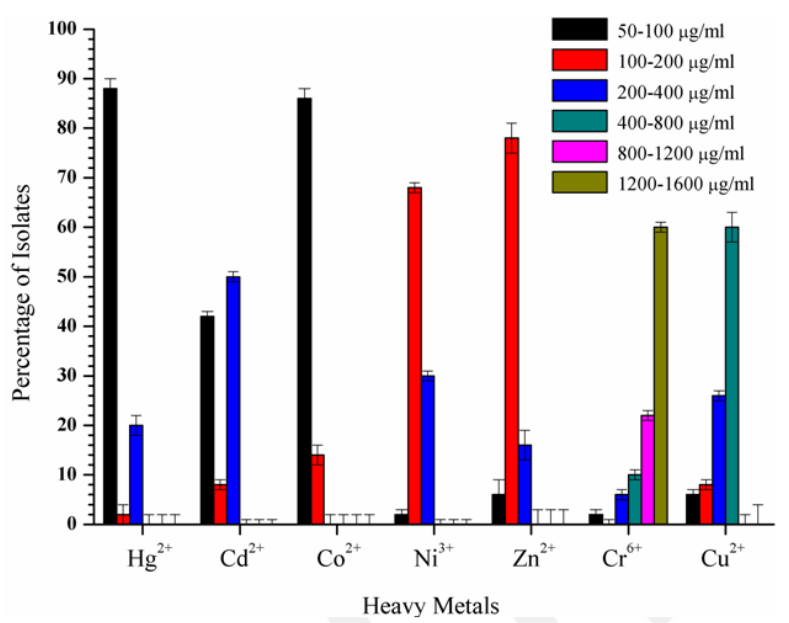

Fig. 3: Gram negative isolates showing various ranges of MIC of metal from site 2).

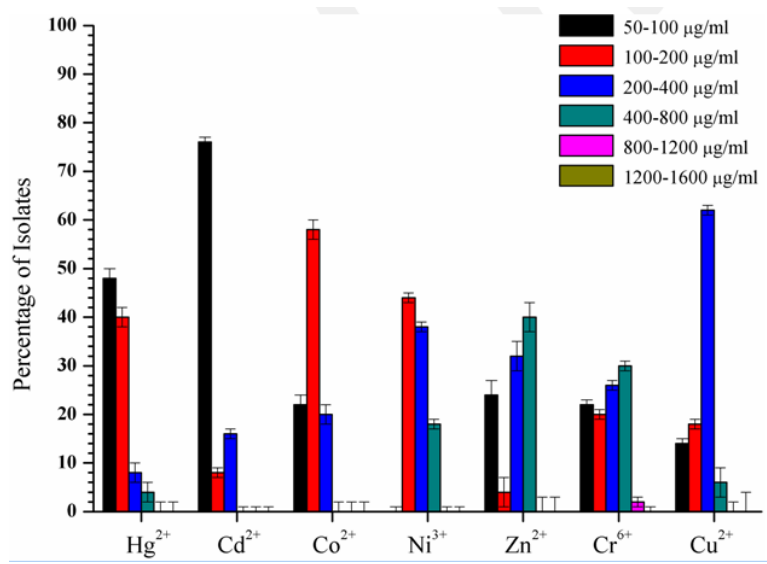

Fig. 4: Gram negative isolates showing various ranges of MIC of metal from site 3).

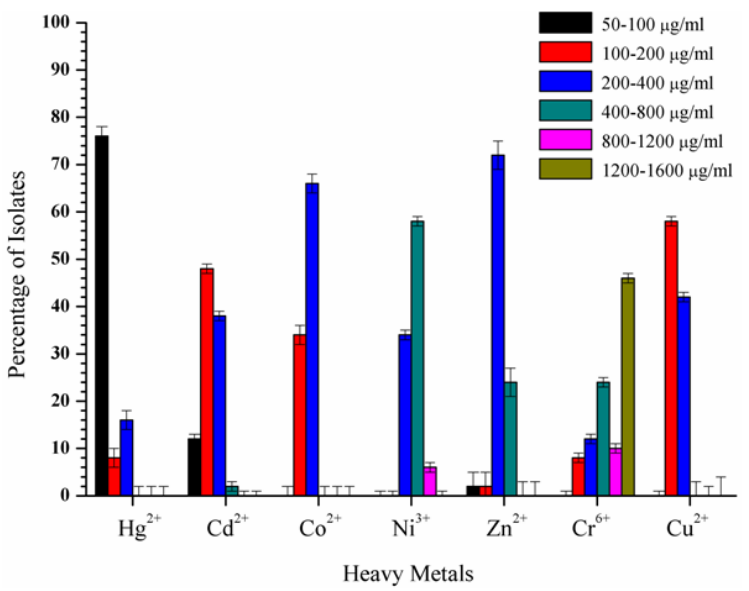

Fig. 5: Gram negative isolates showing various ranges of MIC of metal from site 4).

Majority of isolates from all the sites exhibited resistance to multiple metals (Tables 3, 4 and 5). Maximum $42 \%$ and $38 \%$ of 
the isolates showed 6 and 5 metal resistance pattern at a time in five different combinations in site-I. Whereas, in site-II $36 \%$ and $34 \%$ of isolates exhibited resistance pattern among 4 and 6 metal at a time in two and three different combinations.

Table 3: Multi-metal resistance pattern in 50 gram negative bacterial isolates from SGPGI hospital (Treated).

\begin{tabular}{ccccc}
\hline $\begin{array}{c}\text { No of } \\
\text { Metal }\end{array}$ & Resistance Pattern & Isolates & $\begin{array}{c}\text { Percentage } \\
(\%)\end{array}$ & $\begin{array}{c}\text { MMR } \\
\text { Index }\end{array}$ \\
\hline \multirow{2}{*}{3} & $\begin{array}{c}\mathrm{Cu}, \mathrm{Ni}, \mathrm{Zn} \\
\mathrm{Cr}, \mathrm{Cu}, \mathrm{Zn} \\
\mathrm{Cr}, \mathrm{Ni}, \mathrm{Zn}\end{array}$ & $\begin{array}{c}1 \\
2\end{array}$ & 8 & 0.10 \\
\hline \multirow{2}{*}{4} & $\mathrm{Cr}, \mathrm{Cu}, \mathrm{Ni}, \mathrm{Zn}$ & 16 & 36 & \multirow{2}{*}{0.03} \\
\hline \multirow{5}{*}{5} & $\mathrm{Cr}, \mathrm{Cu} \mathrm{Ni}, \mathrm{Cd}$ & 2 & & \\
& $\mathrm{Cr}, \mathrm{Cu}, \mathrm{Ni}, \mathrm{Cd}, \mathrm{Zn}$ & 3 & & 0.06 \\
& $\mathrm{Co}, \mathrm{Cr}, \mathrm{Cu}, \mathrm{Ni}, \mathrm{Zn}$ & 1 & 22 & \multirow{2}{*}{0.05} \\
\hline \multirow{2}{*}{6} & $\mathrm{Co}, \mathrm{Cr}, \mathrm{Cu}, \mathrm{Ni}, \mathrm{Cd}$ & 1 & & \\
\hline
\end{tabular}

Table 4: Multi-metal resistance pattern in 50 gram negative bacterial isolates from SGPGI hospital (Untreated).

\begin{tabular}{|c|c|c|c|c|}
\hline $\begin{array}{l}\text { No of } \\
\text { Metal }\end{array}$ & Resistance Pattern & Isolates & $\begin{array}{c}\text { Percentage } \\
(\%)\end{array}$ & $\begin{array}{l}\text { MMR } \\
\text { Index }\end{array}$ \\
\hline \multirow{2}{*}{2} & $\mathrm{Cr}, \mathrm{Ni}$ & 2 & \multirow{2}{*}{6} & \multirow{2}{*}{0.09} \\
\hline & $\mathrm{Ni}, \mathrm{Zn}$ & 1 & & \\
\hline \multirow{6}{*}{3} & $\mathrm{Cu}, \mathrm{Ni}, \mathrm{Zn}$ & 1 & \multirow{6}{*}{12} & \multirow{6}{*}{0.07} \\
\hline & $\mathrm{Cr}, \mathrm{Cu}, \mathrm{Zn}$ & 1 & & \\
\hline & $\mathrm{Cr}, \mathrm{Ni}, \mathrm{Zn}$ & 1 & & \\
\hline & $\mathrm{Co}, \mathrm{Cu}, \mathrm{Ni}$ & 1 & & \\
\hline & $\mathrm{Cr}, \mathrm{Cd}, \mathrm{Zn}$ & 1 & & \\
\hline & $\mathrm{Cr}, \mathrm{Co}, \mathrm{Ni}$ & 1 & & \\
\hline \multirow{6}{*}{4} & $\mathrm{Cr}, \mathrm{Cu}, \mathrm{Ni}, \mathrm{Zn}$ & 1 & \multirow{6}{*}{16} & \multirow{6}{*}{0.07} \\
\hline & $\mathrm{Cr}, \mathrm{Ni}, \mathrm{Cd}, \mathrm{Zn}$ & 1 & & \\
\hline & $\mathrm{Co}, \mathrm{Cu}, \mathrm{Ni}, \mathrm{Zn}$ & 2 & & \\
\hline & $\mathrm{Hg}, \mathrm{Cr}, \mathrm{Ni}, \mathrm{Zn}$ & 1 & & \\
\hline & $\mathrm{Hg}, \mathrm{Co}, \mathrm{Cu}, \mathrm{Ni}$ & 2 & & \\
\hline & $\mathrm{Co}, \mathrm{Cr}, \mathrm{Cu} . \mathrm{Ni}$ & 1 & & \\
\hline \multirow{6}{*}{5} & Co. $\mathrm{Cr}, \mathrm{Cu}, \mathrm{Ni}, \mathrm{Zn}$ & 11 & \multirow{6}{*}{36} & \multirow{6}{*}{0.03} \\
\hline & $\mathrm{Hg}, \mathrm{Cr}, \mathrm{Cu}, \mathrm{Ni}, \mathrm{Zn}$ & 1 & & \\
\hline & $\mathrm{Hg}, \mathrm{Co}, \mathrm{Cu}, \mathrm{Ni}, \mathrm{Zn}$ & 2 & & \\
\hline & $\mathrm{Hg}, \mathrm{Co}, \mathrm{Cu}, \mathrm{Ni}, \mathrm{Cd}$ & 1 & & \\
\hline & $\mathrm{Hg}, \mathrm{Co}, \mathrm{Cr}, \mathrm{Cu}, \mathrm{Ni}$ & 2 & & \\
\hline & $\mathrm{Co}, \mathrm{Cr}, \mathrm{Cu}, \mathrm{Ni}, \mathrm{Cd}$ & 1 & & \\
\hline \multirow{3}{*}{6} & $\mathrm{Hg}, \mathrm{Co}, \mathrm{Cr}, \mathrm{Cu}, \mathrm{Ni}, \mathrm{Zn}$ & 10 & \multirow{3}{*}{26} & \multirow{3}{*}{0.06} \\
\hline & $\mathrm{Hg}, \mathrm{Co}, \mathrm{Cr}, \mathrm{Cu}, \mathrm{Ni}, \mathrm{Cd}$ & 1 & & \\
\hline & $\mathrm{Co}, \mathrm{Cr}, \mathrm{Cu}, \mathrm{Ni}, \mathrm{Cd}, \mathrm{Zn}$ & 2 & & \\
\hline 7 & $\mathrm{Hg}, \mathrm{Co}, \mathrm{Cr}, \mathrm{Cu}, \mathrm{Ni}, \mathrm{Cd}, \mathrm{Zn}$ & 2 & 4 & 0.5 \\
\hline
\end{tabular}

Table 5: Multi-metal resistance pattern in 50 gram negative bacterial isolates from RML hospital (Untreated).

\begin{tabular}{lllll}
\hline $\begin{array}{l}\text { No. of } \\
\text { Metal }\end{array}$ & \multicolumn{1}{c}{ Resistance Pattern } & Isolates & $\begin{array}{c}\text { Percentage } \\
(\%)\end{array}$ & $\begin{array}{c}\text { MMR } \\
\text { index }\end{array}$ \\
\hline 5 & $\mathrm{Co}, \mathrm{Cu}, \mathrm{Ni}, \mathrm{Cd}, \mathrm{Zn}$ & 1 & 2 & 0.71 \\
\hline 6 & $\mathrm{Hg}, \mathrm{Co}, \mathrm{Cr}, \mathrm{Cu}, \mathrm{Ni}, \mathrm{Zn}$ & 2 & 60 & 0.02 \\
& $\mathrm{Co}, \mathrm{Cr}, \mathrm{Cu}, \mathrm{Ni}, \mathrm{Cd}, \mathrm{Zn}$ & 28 & & 0.05 \\
\hline 7 & $\mathrm{Hg}, \mathrm{Co}, \mathrm{Cr}, \mathrm{Cu}, \mathrm{Ni}, \mathrm{Cd}, \mathrm{Zn}$ & 19 & 38 & \\
\hline
\end{tabular}

In addition, of $36 \%$ and $26 \%$ isolates showed metal resistance pattern among five and six metal at a time in five and three different combinations from site-III while $60 \%$ and $38 \%$ of the isolates exhibited metal resistance pattern among 6 and 7 metals at a time in two and one combination in site-4, respectively. Resistance potential of the isolates was also evaluated in terms of multiple metal resistance indexes. A varied trend of MMR Index was observed among the isolates from the four different sampling sites. Low and high risk MMR were recorded among the Gram negative bacterial isolates from the hospital wastewater. MMR range $0.03-0.42,0.03-0.10,0.03-0.5$ and $0.05-0.71$ were recorded among the isolates from site I (untreated), site II (treated), site III and site IV (untreated), respectively.

\section{DISCUSSION}

Metal resistance is a common process in many microorganisms that deal with toxic compounds in their habitats. In the last few years, metal resistance has increased our knowledge about the cellular mechanisms involved in metal resistance. Mercury resistance has been described in a number of bacterial species (Nakamura and Silver, 1994; Mergeary et al., 2003). In Present study, all the isolates were tested for viable count and their resistance against certain metals $\left(\mathrm{Hg}^{2+}, \mathrm{Cd}^{2+}, \mathrm{Cu}^{2+}, \mathrm{Zn}^{2+}, \mathrm{Ni}^{2+}\right.$, $\mathrm{Co}^{2+}$ and $\left.\mathrm{Cr}^{3+}\right)$. The viable count of gram negative bacteria in different concentrations of metal ranged from $11.03 \times 10^{5}-8.0 \times 10^{2}$, $12.02 \times 10^{5}-1.0 \times 10^{2}, \quad 12.33 \times 10^{5}-5.0 \times 10^{2}$ and $4.01 \times 10^{4}-1.0 \times 10^{2}$ $\mathrm{cfu} / \mathrm{ml}$ of water in site I, II, III and IV, respectively. Out of 50 Gram negative bacteria isolated from all sites, $42 \%$ were found to be resistant to six metal ions at a time in five different combinations, while only $2.0 \%$ of the isolates were resistant to three metal ions at a time. $36 \%$ resistance were found in four and five metals at a time in two and five different combinations in both site II and III, respectively. Maximum $60 \%$ of the isolates were found to be resistance to six metals at a time in two different combinations and $38 \%$ of the isolates were resistance in seven metals at a time in site IV (Table 3, 4, 5). The frequency of metal resistance in the present study is comparable to those reported elsewhere (Malik and Jaiswal, 2000; Ansari et al., 2008; Alam and Imran, 2014). Malik and Aleem, (2010) reported that the majority of bacterial isolates from metal contaminated soil showed resistance to multiple metal ions. $20.8 \%$ of the pseudomonas isolates were resistant to eight metal ions at a time, while $12.5 \%$ of the isolates from groundwater irrigated soil were resistant to five metal ions at a time in two different combinations. Similar observations have also been reported earlier (Appanna et al., 1996; Malik et al., 2008; Wei et al., 2009). Present results exhibited a high incidence of metal resistance in the isolates from untreated wastewater as compared to treated wastewater (Table 2). Sabry et al, (1997) isolated heterotrophic aerobic metal-resistant bacterial communities from marine water and reported that great portion of the isolates were resistant to lead (94\%), nickel (40\%), arsenate (35\%) and copper (22\%). Similarly, Shakoori and Muneer, (2002) also reported that bacteria from wastewater origin exhibited resistance against $\mathrm{Ag}^{2+}(280-350 \mu \mathrm{g} / \mathrm{ml}), \mathrm{Co}^{2+}(200-420 \mu \mathrm{g} / \mathrm{ml})$, $\mathrm{Cr}^{6+}(280-400 \mu \mathrm{g} / \mathrm{ml}), \mathrm{Cd}^{2+}(250-350 \mu \mathrm{g} / \mathrm{ml}), \mathrm{Hg}^{2+}(110-200$ $\mu \mathrm{g} / \mathrm{ml}), \mathrm{Mn}^{2+}(300-380 \mu \mathrm{g} / \mathrm{ml}), \mathrm{Pb}^{2+}(300-400 \mu \mathrm{g} / \mathrm{ml}), \mathrm{Sn}^{2+}(480-$ $520 \mu \mathrm{g} / \mathrm{ml})$ and $\mathrm{Zn}^{2+}(300-450 \mu \mathrm{g} / \mathrm{ml})$. In the present study, maximum $88 \%, 76 \%$ and $72 \%$ bacterial isolates from hospital wastewater exhibited MIC value of $50-100 \mu \mathrm{g} / \mathrm{ml}$ for $\mathrm{Hg}^{2+}$ while 
$60 \%$ and $30 \%$ of the isolates showed their MIC range 1200-1600 $\mu \mathrm{g} / \mathrm{ml}$ against $\mathrm{Cr} 3$ and $\mathrm{Cu} 2+$ in entire site tested respectively. High levels of resistance were found in $\mathrm{Cu}^{2+}, \mathrm{Zn}^{2+} \mathrm{Cr} 3+, \mathrm{Ni}^{2+}$ and $\mathrm{Co}^{2+}$ respectively. This coherent with other reports where author have found that the multi-resistant strains had higher MIC values compare to the sensitive ones (Karbasizaed et al., 2003; Vajiheh and Naser, 2003; Basu et al., 1997). We also determined the MMR index of Gram negative bacterial isolates from all sampling sites. Isolates exhibited a variation in their MMR index based on sampling sites. Low and high risk MMR were recorded among the Gram negative bacterial isolates from the hospital wastewater. MMR index is ranges between 0.03-0.71 among the isolates from entire sites tested.

\section{CONCLUSION}

Our observations are contributing to the understanding of metal tolerant among Gram negative bacteria from aquatic environment and underline the importance of describing the succession of bacterial populations indigenously present in such environment due to contamination events. Bacterial resistance to antibiotics and heavy metals is an increasing problem in today's society. Microbes have adapted to tolerate the presence of metals or can even use them to grow. Thus, a number of interactions between microbes and metals have important environmental and health implications. Accordingly, considerable variation in MIC of untreated and treated isolates has been found. Multiple resistances in untreated isolates have also been observed, indicating public health concern. Therefore, there is urgent need of detoxification of untreated sites.

\section{ACKNOWLEDGMENT}

We are thankful to Prof. S.W. Akhtar, Vice Chancellor, Integral University, for providing the necessary facility to conduct this research. The authors also thank the HOD, Department of Biosciences and Bioengineering, Integral University, Lucknow for guidance and their cooperation with regard the research work.

\section{Financial support and sponsorship: NIL.}

Conflict of Interests: There are no conflicts of interest.

\section{REFERENCES}

Rutala, WA \& Mayhall CG. Medical waste. Infect Control Hosp Epidemiol 1992; 13:38-48.

Blumenthal, UJ, Cifuentes E, Bennett S, Quigley M, RuizPalacios G. The risk of enteric infections associated with wastewater reuse: the effect of season and degree of storage of wastewater. Trans $\mathrm{R}$ Soc Trop Med Hyg 2001; 95:131-7.

Roane, T.M, Kellogg, S T. Characterization of bacterial communities in heavy metal contaminated soils. Can J Microbiol 1996; 42: 593-603.

Ren, W X, Li, P J, Geng, Yand Li XJ. Biological leaching of heavy metals from a contaminated soil by Aspergillus niger. J. Hazardous materials 2009; 167: 164-169.
Hughes M N, Poole, R K. The functions of metals in microorganisms. In: (eds). Metals and Microorganisms. Chapman and Hall, London 1989; p. 1-38.

Mindlin S, Kholodii G, Gorlenko Z, Minakhina S, Minakhin L, Kalyaeva E, Kopteva A, Petrova M, Yurieva O, Nikiforov V. Mercury resistance transposons of Gram-negative environmental bacteria and their classification. Res. Microbiol 2001; 152: 9, 811-822.

Silver S, Misra T K. Plasmid-mediated heavy metal resistances. Annu. Rev. Microbiol 1988; 42: 717-743.

Foster, T J. Plasmid-determined resistance to antimicrobial drugs and toxic metal ions in bacteria. Microbiol. Rev 1983; 47 (3): 361409.

McIntosh D, Cunningham M Ji B, Fekete FA, Parry EM, Clark SE, Zalinger ZB, Gilg IC, Danner GR, Johnson KA, Beattie M, Ritchie R..Transferable, multiple antibiotic and mercury resistance in Atlantic Canadian isolates of Aeromonas salmonicida subsp. salmonicida is associated with carriage of an IncA/C plasmid similar to the Salmonella enterica plasmid pSN254. J. Antimicrob. Chemother 2008; 61 (6): 12211228.

Viti C, Pace A \& Giovannetti L.. Characterization of Cr (VI) resistant bacteria isolated from chromium-contaminated soil by tannery activity. Current Microbiology 2003; 46: 1-5.

Bell J B, Elliot G E and Smith D E. Influence of sewage treatment and urbanization on selection of multiple faecal coliform populations. Appl. Environ. Microbiol 1983; 46: 227-232.

Nies D H. Microbial heavy metal resistance. Applied Microbiology and Biotechnology 1999; 51: 730-750.

Lösch L S, Alonso J M, Merino L A. Occurrence of antimicrobial-resistant Enterobacteriaceae in water from different sources in a subtropical region of Argentina. Revista Ambi-Água, Taubaté 2008; 2: $28-36$

Cappuccino J G, Sherman N. Inc California America. 1995

Malik A \& Jaiswal R, World J Microbiol Biotechnol 2000;

$16: 177$.

Nakamura K and Silver S. Molecular analysis of mercury resistant Bacillus isolates from sediment of Minamata Bay Japan. Appl. environ Microbiol 1994; 60: 4596-4599.

Mergeary M., Monchy S., Vallaeys T., Auquier V., Benotmane A., Bertin P., Taghavi S, Dunn J, Vander Lelie D, Wattiez R. Metal Resistance in Candida Biofilms. Fems Microbiol 2003; 9: 1181-1191.

Malik A and Jaiswal R. Metal resistance in Pseudomonas strains isolated from soil treated with industrial wastewater. World Journal of Microbiology and Biotechnology 2000; 16: 177-182.

Ansari M I, Grohmann E, Malik A. Conjugative plasmids in multi-resistant bacterial isolates from Indian soil. Journal of Applied Microbiology 2008; 104: 1774-1781.

Alam M, Imran M. Multiple antibiotic resistance in metal tolerant E. coli from hospital wastewater Bioinformation 2014.; 10 (5) : 000-000

Malik A and Aleem A. Incidence of metal and antibiotic resistance in Pseudomonas spp. from the river water, agricultural soil irrigated with wastewater and groundwater. Environ Monit Assess 2010; 10:1007. 10661-010-1690-2.

Appanna, V D, Gazso LG, Pierre S M. Multiple metal tolerance in Pseudomonas fluorescens and its biotechnological significance. Journal of Biotechnology 1996; 52: 75-80.

Malik A, Celik E K, Bohn C, Böckelmann U, Knobel K \& Grohmann E. Detection of conjugative plasmids and antibiotic resistance genes in anthropogenic soil from Germany and India. FEMS Microbiology Letters 2008; 279: 207-216.

Wei G, Fan L, Zhu W, Fu Y, Yu J. and Tang M. Isolation and characterization of the heavy metal resistant bacteria Lespedeza cuneata CCNWRS33 2 isolated from root nodule of in gold mine tailings in China. J. Hazardous Materials 2009; 162, 50-56.

Sabry S A, Ghozalan H A, Abou-Zeid D M. Metal tolerance and antibiotic resistance patterns of a bacterial population isolated from sea water. Journal of Applied Microbiology 1997; 62: 245-252. 
Shakoori A R and Muneer B. Copper-resistant bacteria from industrial effluents and their role in remediation of heavy metals in wastewater. Folia Microbiologica (Praha) 2002; 47: 43-50.

Karbasizaed V, Badami N, Emtiazi G. Antimicrobial, heavy metal resistance and plasmid profile of coliforms isolated from nosocomial infections in a hospital in Isfahan, Iran. African Journal of Biotechnology 2003; 2: (10). 379-383.

Vajiheh, K and Naser, B. Antimicrobial, heavy metal resistance and plasmid profile of coliforms isolated from nosocomial infection in a hospital in Isfahan, Iran. African J Biotechnol 2003; 2: 379-383.

Basu M, Bhattacharya S, Paul A K. Isolation and characterization of chromium-resistant bacteria from tannery effluents. Bull. Environ. Contam. Toxicol 1997; 58 (4): 535-542.

How to cite this article:

Alam M, Imran M. Metal tolerance analysis of Gram negative bacteria from hospital effluents of Northern India. J App Pharm Sci, 2017; 7 (04): 174-180. 